\title{
Association of British Clinical Diabetologists (ABCD) and Diabetes UK joint position statement and recommendations for non-diabetes specialists on the use of sodium glucose co-transporter 2 inhibitors in people with type 2 diabetes (January 2021)
}

\author{
Authors: Umesh Dashora, ${ }^{\mathrm{A}}$ Robert Gregory, ${ }^{\mathrm{B}}$ Peter Winocour, ${ }^{\mathrm{C}}$ Ketan Dhatariya, ${ }^{\mathrm{D}}$ Susannah Rowles, ${ }^{\mathrm{E}}$ \\ Andrew Macklin, ${ }^{\mathrm{F}}$ Gerry Rayman, ${ }^{\mathrm{G}}$ Dinesh Nagi, ${ }^{\mathrm{H}}$ Katie Whitehead, ${ }^{\mathrm{I}}$ Hannah Beba ${ }^{\mathrm{J}}$ Parijat $\mathrm{De}^{\mathrm{K}}$ and \\ Dipesh C Patel; ' on behalf of the ABCD executive committee and Diabetes UK
}

Sodium glucose co-transporter 2 (SGLT2) inhibitors are now an established class of medications for the treatment of type 2 diabetes (T2D), no longer reserved for use by specialists in diabetes. They are being used increasingly for their cardiac and renal benefits by primary care, cardiology and renal teams for indications in parallel with diabetes care as part of holistic management. This guidance provides essential information on SGLT therapy, including the main advantages and the important risks of which healthcare professionals should be aware.

Authors: ${ }^{A}$ consultant (diabetes), honorary reader, deputy sub-dean and meeting secretary ABCD, Conquest Hospital, St Leonards-on-Sea, UK; ${ }^{B}$ consultant physician, University Hospitals of Leicester NHS Trust, Leicester, UK; ${ }^{C}$ consultant physician and clinical director, East and North Hertfordshire NHS Trust, Stevenage, UK; ${ }^{\text {D }}$ consultant physician and honorary professor, Norfolk and Norwich University Hospitals NHS Foundation Trust, Norwich, UK; E consultant diabetologist, Pennine Acute Hospitals NHS Trust, Manchester, UK; ${ }^{\text {F }}$ consultant diabetologist, Dorset County Hospital NHS Foundation Trust, Dorchester, UK; ${ }^{G}$ consultant diabetologist, honorary professor and clinical lead for diabetes GIRFT, East Suffolk and North East Essex NHS Foundation Trust, Ipswich, UK; ${ }^{H}$ honorary consultant in diabetes and endocrinology, and Ex Officio (Chair) ABCD, Pinderfields Hospital, Wakefield, UK; ' practice nurse, The Hollies Medical Centre, Sheffield, UK; ' senior clinical pharmacist in diabetes and endocrinology, and cochair diabetes and endocrinology committee UKCPA, County Durham and Darlington NHS Foundation Trust, Durham, UK; ${ }^{K}$ consultant physician in diabetes and endocrinology, Sandwell and West Birmingham Hospital NHS Trust, Sandwell, UK; ' 'consultant physician in diabetes and endocrinology, and honorary associate professor, University College London, London, UK
KEYWORDS: SGLT2 inhibitors, type 2 diabetes, non-diabetes specialists, $A B C D$, Diabetes UK

DOI: $10.7861 /$ clinmed.2021-0045

\section{What is new?}

The American Diabetes Association / European Association for the Study of Diabetes (ADA/EASD) consensus report 2019 update recommends the use of sodium glucose co-transporter 2 (SGLT2) inhibitors with proven cardiovascular and renal benefits as a second-line therapy independent of glycated haemoglobin (HbA1c) target after metformin when there is a need to address atherosclerotic cardiovascular disease (previous myocardial infarction, angina or peripheral vascular disease), heart failure, renal impairment, excess body weight or risk of hypoglycaemia. ${ }^{1}$

SGLT2 inhibitors are being increasingly used in primary care and by non-diabetes specialist teams.

SGLT2 inhibitors are commonly associated with genital mycotic infections. They are also associated with a small increase in risk of diabetic ketoacidosis in people with type 2 diabetes (T2D).

Here, we summarise available evidence and provides guidance on how to reduce adverse effects of SGLT2 therapy and manage them when they occur.

What are the types of diabetes?

Type 1 diabetes (T1D) is caused by the destruction of the insulin producing beta cells in the pancreas. ${ }^{2}$ People with this type of diabetes rapidly become dependent on insulin for survival. Insulin supplementation should never be stopped even for a short period in absolute insulin deficiency as this risks diabetic ketoacidosis (DKA)

T2D develops because of a mix of resistance to insulin action and deficiency of insulin secretion with variable genetic susceptibility combined with environmental factors (like sedentary lifestyle or obesity). Pharmacotherapy is often required if diet and exercise 
alone are not sufficient to control diabetes. Metformin is the firstline therapy. SGLT2 inhibitors or glucagon-like peptide 1 (GLP-1) analogues with proven cardiovascular benefit are increasingly being used as a second-line therapy based on recent clinical trial results. As diabetes progresses, combination therapy may be needed and insulin may be required to manage hyperglycaemia. Some people with apparent T2D may actually have a slowly progressing T1D (latent autoimmune diabetes in adults (LADA) with positive insulin auto-antibodies) or damage to their entire pancreas (type $3 c$ diabetes). These people are at higher risk of DKA. Other groups with T2D are also ketosis-prone for unknown reasons. ${ }^{3} \mathrm{~T} 2 \mathrm{D}$ may present with DKA in some people (particularly of Black ethnicity) but these people often do not require insulin in the long term. In addition, people with T2D and severe illness can have an increase in insulin requirement that precipitates DKA

\section{How is diabetes diagnosed?}

Diabetes is diagnosed by a single fasting plasma glucose $\geq 7 \mathrm{mmol} / \mathrm{L}$ or an $\mathrm{HbA} 1 \mathrm{c} \geq 48 \mathrm{mmol} / \mathrm{L}$ in the presence of osmotic symptoms (polydipsia, polyuria, tiredness or thirst) or the same levels on two different occasions in the absence of symptoms. ${ }^{2}$ Up to $90 \%$ of people with T1D have positive auto-antibodies at presentation. Serum or urine C-peptide is initially low, but present. This declines with time, such that C-peptide becomes undetectable in most by 5 years. C-peptide production also decreases in T2D, although much more slowly.

\section{The unmet need in the treatment of people with T2D}

Up to $50 \%$ of people with T2D die from cardiovascular disease with the increased mortality strongly attributed to diabetic kidney disease. ${ }^{4,5}$ Therefore, drugs with robust evidence of reducing cardiovascular and renal morbidity and mortality in people with T2D need to be used widely. Additionally, a number of treatment options for T2D are associated with unwanted side effects (such as hypoglycaemia and weight gain). Efficacious drugs not associated with such side effects are also highly desirable. ${ }^{6}$

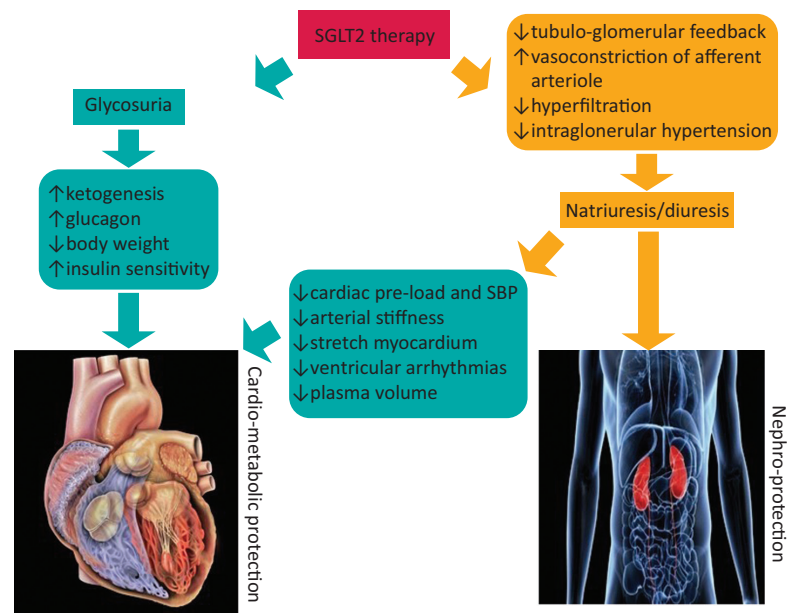

Fig 1. Cardiovascular and renal effects of sodium glucose co-transporter 2 inhibitors. ${ }^{7}$ SBP $=$ systolic blood pressure; SGLT2 = sodium glucose cotransporter 2

\section{What are SGLT2 inhibitors?}

SGLT2 inhibitors are an established class of medications for the treatment of diabetes which act by preventing the absorption of glucose and sodium, mainly from the proximal renal tubule in the kidney. Glucose and sodium are, therefore, lost in urine. People do not become hyponatraemic (unless on diuretics as well) as most of the sodium is reabsorbed in the distal tubule. This effect results in decreasing the blood glucose level, weight loss, an osmotic diuresis and a drop in blood pressure (Fig 1). ${ }^{7}$ These drugs have been licensed and used widely in people with T2D and have shown significant cardiovascular and kidney benefits in different subsets of this group of patients. ${ }^{8}$

\section{What are the benefits of using SGLT2 inhibitors?}

Clinical trials using SGLT2 inhibitors have provided strong evidence in the trial population for reduced risk of major cardiovascular, renal and heart failure events (Tables 1 and 2: evidence differs for different agents within the class) in addition to reducing $\mathrm{HbA} 1 \mathrm{c}$ by up to $10 \mathrm{mmol} / \mathrm{mol}(1.0 \%)$ depending upon the initial $\mathrm{HbA1c}$. These medications are associated with a low incidence of hypoglycaemia, weight loss of up to $3 \mathrm{~kg}$ of body weight and a reduction in systolic blood pressure of approximately $3-5 \mathrm{mmHg}$. It is important to select the right patient for SGLT2 inhibitors in order to achieve health benefits (Box 1) without precipitating complications like DKA (Table 3).

\section{What to do when initiating SGLT2 inhibitors: information for the patient}

Treatment with SGLT2 inhibitors should be initiated only after ensuring adequate understanding of the person in the following aspects.

$>$ Discuss individualised benefits of taking SGLT2 Inhibitors.

$>$ Common side effects (Table 4).

$>$ Uncommon side effects (Table 4).

$>$ Foot care (Table 4)

$>$ Drink plenty of fluids to avoid dehydration unless you have been told to restrict fluids by your healthcare professionals due to heart or kidney problems or some other reason.

\section{How to reduce the risk? Educate the patient about sick day guidance and DKA}

When a person with diabetes is not well and is unable to eat and drink as normal, some simple rules can prevent further deterioration or DKA. ${ }^{18}$

> If ill with diarrhoea, vomiting or fever and unusual drowsiness, stop SGLT2 inhibitors and don't restart until feeling better and eating/drinking fluids normally.

$>$ When people with diabetes who take insulin are not able to eat, it may be possible for them to consume half a glass of milk or fruit juice or yogurt or soup (not clear soup) in place of meals and follow individualised advice from their healthcare professionals about what dose of insulin to take.

$>$ Drink plenty of water / sugar-free fluid to avoid dehydration for up to 24 hours.

> Seek medical advice if particularly unwell with infection or illness. 


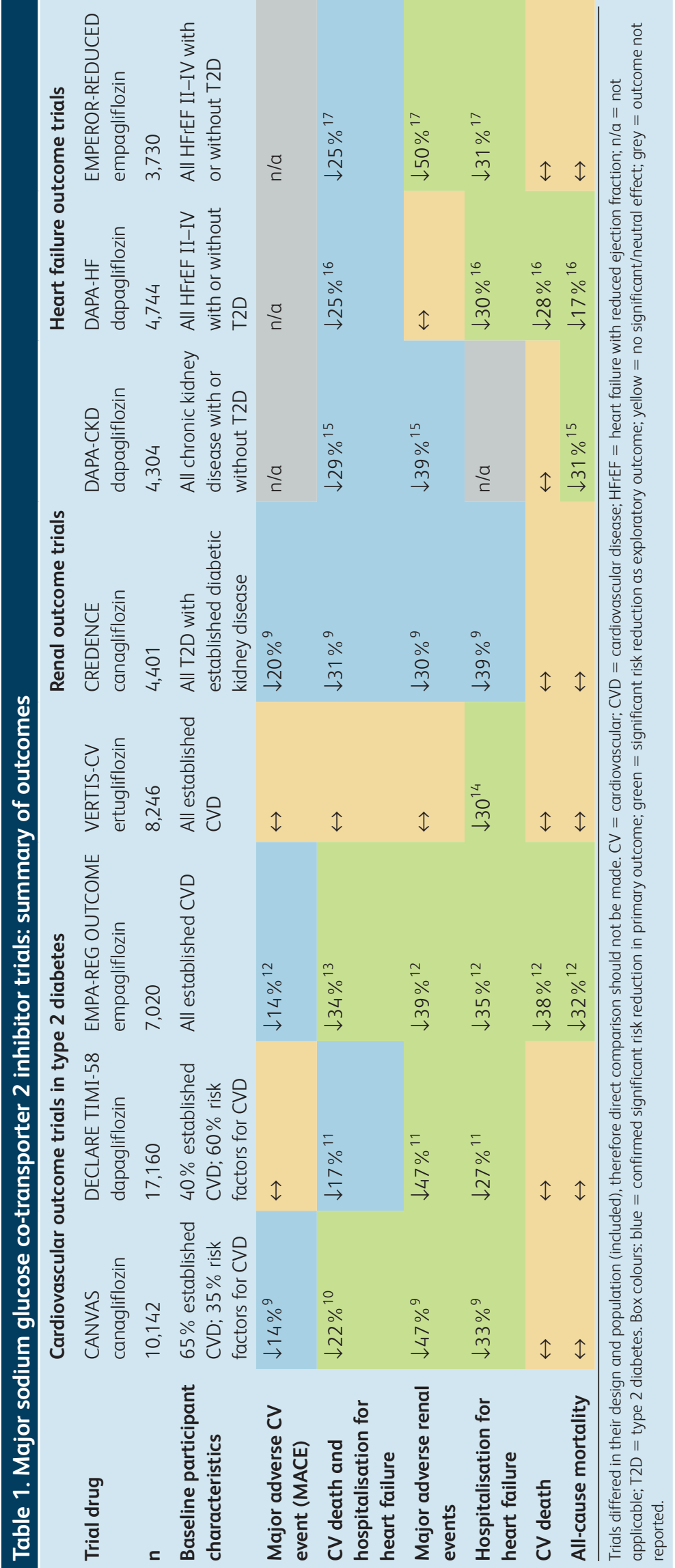




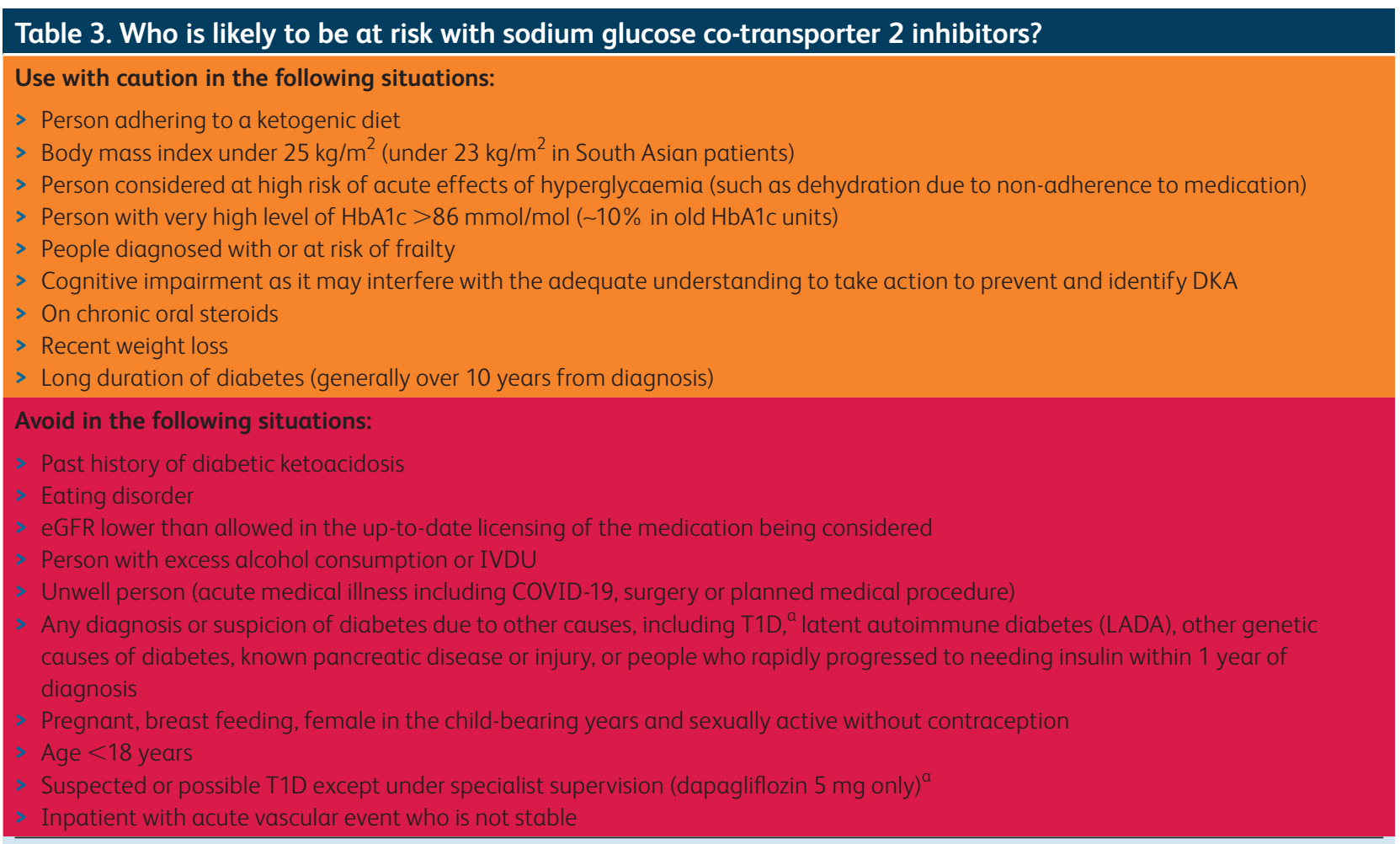

Seek advice from the local diabetes team if unsure about the benefits and risks. ${ }^{a}$ Only dapagliflozin $5 \mathrm{mg}$ is licensed for use in T1D in certain circumstances and should be initiated and supervised by a specialist. Sotagliflozin (SGLT1+2 inhibitor) $200 \mathrm{mg}$ is also approved by NICE for similar circumstances but not currently available in the UK. eGFR = estimated glomerular filtration rate; DKA = diabetic ketoacidosis; HbA1c = glycated haemoglobin; IVDU = intravenous drug users; NICE $=$ National Institute for Health and Care Excellence; T1D = type 1 diabetes.

\section{Table 4. Adverse reactions to sodium glucose co-transporter 2 inhibitors ${ }^{9,11,12,19-21}$}

\section{Adverse reaction}

Genital mycotic infections Frequency

Common / very common

Increased urination

Common

Urinary tract infections

Common

Volume depletion side effects

(thirst, postural dizziness,

hypotension and dehydration)

Diabetic ketoacidosis

Amputation
Common/uncommon (varies with agent)

Rare: $<1 / 1,000$ in SmPC (between 0 and 2 additional events per 1,000 person-years in RCTs); real life events may be higher

Uncommon: event rate $<1 / 100$ in SmPC (between 0 and 3 additional events per 1,000 person-years in RCTs); ${ }^{a}$ real-life events may be lower

\section{Notes}

Both male and female. Provide genital hygiene advice. Most initial cases can be treated with topical antifungals and won't recur. Consider reviewing therapy if recurrent infections.

Increased frequency and/or increased volume.

In recent large trials, any increase in risk was small and non-significant.

Caution in frail/elderly. Consider measuring blood pressure in lying and standing position in those at risk of fall and those on diuretics

Inform and advise patients and healthcare professionals about how to prevent diagnose and treat DKA

Encourage routine preventative foot care; regular foot checking

Advise patients to report wounds, discoloration or tender/ painful feet

Consider stopping therapy if significant foot problems arise (such as infection or skin ulcers)

${ }^{a}$ Excess amputations were only seen in one study; subsequent studies with the same SGLT2 inhibitor and others have not confirmed a significant increase; any risk is likely to be very low. Necrotising fasciitis (Fournier's gangrene): post-marketing reports have been reported (six yellow card reports in $>500,000$ patient-years. Recent large trials have not shown any increase in risk. Patients should be advised to seek urgent medical attention if they experience fever/malaise along with pain, tenderness or redness in the genital or perineal area. ${ }^{22} \mathrm{DKA}=$ diabetic ketoacidosis; $\mathrm{SmPC}=$ summary of product characteristics. Frequency: very common $=\geq 1 / 10$; common $=\geq 1 / 100$ to $<1 / 10$; uncommon $=\geq 1 / 1,000$ to $<1 / 100$; rare $=\geq 1 / 10,000$ to $<1 / 1,000$. 
> As ketones may lead to worse surgical outcome, SGLT inhibitors should be stopped 48 hours before elective surgery. However, ketones should be tested for in the presence of symptoms suggestive of DKA even after interruption of SGLT2 inhibitors. In addition, ketones should be periodically checked (as required) if the admission is for emergency surgery. SGLT2 inhibitors can be restarted 24 hours after resuming normal oral intake.

> Useful information has been produced by national cardiology, renal and metabolic associations which can be downloaded and shared with people with diabetes. ${ }^{24}$

\section{What to do when initiating SGLT2 inhibitors: information for the prescriber}

When SGLT2 inhibitors are started, the healthcare provider should document completion of the education session with the person with diabetes and offer some advice on who to contact if the person taking the SGLT2 inhibitor is not feeling well. Glucose lowering medications that may cause hypoglycaemia, such as insulin and sulphonylureas, should be reviewed and consideration should be given to reduce the dose when SGLT2 inhibitor is started, particularly if the individual's $\mathrm{HbA1} \mathrm{c}$ is at target when the treatment is being initiated. If the insulin requirement reduces considerably, one should be cautious of a higher risk of developing DKA. The healthcare professional should review diuretic and antihypertensive therapy periodically if hypertension improves or if there is postural hypotension.

\section{How to reduce the risk of DKA and manage it? Educate the health professionals}

In patients with diabetes on SGLT2 inhibitors with symptoms suggestive of DKA, ketosis should be confirmed by measuring capillary or venous blood ketone. If the ketone levels are above $1.5 \mathrm{mmol} / \mathrm{L}$, acidosis should be checked by measuring venous bicarbonate $(<18 \mathrm{mmol} / \mathrm{L})$ or venous $\mathrm{pH}(<7.38)$. Blood glucose level in this situation can be normal because SGLT2 inhibitors induce glucose excretion. If DKA is confirmed (ketones $>3.0$ $\mathrm{mmol} / \mathrm{L}$, bicarbonate $<15 \mathrm{mmol} / \mathrm{L}$ and $\mathrm{pH}<7.30$ ), then the severity will be assessed by local criteria and treated according to the Joint British Diabetes Society (JBDS) guidelines or a locally adapted protocol. ${ }^{23}$ Intravenous glucose in addition to fixed rate insulin infusion may be required to avoid hypoglycaemia while treating euglycaemic DKA.

\section{When to stop treatment with SGLT2 inhibitors?}

It is useful to remember that SGLT2 inhibitors may have to be temporarily stopped in situations which can increase the risk of DKA. Healthcare professionals should be especially careful in the following situations. After an episode of DKA the medications should not be restarted in future.

> Suspend SGLT2 inhibitors in the following circumstances:

$>$ acute medical admission including COVID-19

$>$ admission for elective surgery or procedure requiring starvation

$>$ vomiting

$>$ dehydration.

> Restart only AFTER patient has been eating normally for AT LEAST 24 hours AND no longer acutely unwell.

> Alternative diabetes treatment may be required in the interim.
In summary, SGLT2 inhibitors are a major advance in the management of T2D with additional cardiovascular and renal benefits. These drugs are now being commonly used by primary care physicians, cardiologists and nephrologists and some understanding of their potential benefits and possible side effects is critical.

\section{Acknowledgement}

A version of this paper, adapted for a primary care audience, is due to be published in Dashora $U$, Wheatcroft $\mathrm{S}$, Winocour $\mathrm{P}$ et al; on behalf of the CaReMe UK partnership. Take a holistic approach to the management of diabetes and co-morbidities. Guidelines in Practice 2021;24. www.guidelinesinpractice.co.uk/456004.article

\section{References}

1 Buse JB, Wexler DJ, Tsapas A et al. 2019 update to: management of hyperglycemia in type 2 diabetes, 2018. A consensus report by the American Diabetes Association (ADA) and the European Association for the Study of Diabetes (EASD). Diabetes Care 2020;43:487-93.

2 American Diabetes Association. 2. Classification and Diagnosis of Diabetes: Standards of Medical Care in Diabetes-2020. Diabetes Care 2020;43:\$14.

3 Dhatariya KK, Glaser NS, Codner E, Umpierrez GE. Diabetic ketoacidosis. Nat Rev Dis Primers 2020;6:40.

4 World Health Organization. Diabetes: Data and statistics. WHO. www.euro.who.int/en/health-topics/noncommunicable-diseases/ diabetes/data-and-statistics [Accessed 14 January2020].

5 Afkarian M, Sachs M, Kestenbaum B et al. Kidney disease and increased mortality risk. Journal of American Society of Nephrology 2013,24:302-8

6 Bar-Tana J. Type 2 diabetes - unmet need, unresolved pathogenesis, mTORC1-centric paradigm. Rev Endocr Metab Disord 2020;21:613-29.

7 De Block C. SGLT2 inhibitors and GLP-1 receptor agonists: a sound combination. Lancet Diabetes Endocrinol 2018;6:349-52.

8 Loutradis C, Papadopoulou E, Angeloudi E, Karagiannis A, Sarafidis P. The Beneficial Hemodynamic Actions of SGLT2 Inhibitors beyond the Management of Hyperglycemia. Curr Med Chem 2020;27:6682-702.

9 Electronic Medicines Compendium. Invokana $100 \mathrm{mg}$ and $300 \mathrm{mg}$ film-coated tablets. Summary of product characteristics. EMC. www.medicines.org.uk/emc/product/8855 [Accessed 12 November 2020].

10 Neal B, Perkovic V, Mahaffey KW et al. Canagliflozin and cardiovascular and renal events in type 2 diabetes. N Engl J Med 2017:377:644-57.

11 Electronic Medicines Compendium. Forxiga $5 \mathrm{mg}$ and $10 \mathrm{mg}$ film coated tablets. Summary of Product Characteristics. EMC. www. medicines.org.uk/emc/product/2865/smpc [Accessed 12 November 2020].

12 Electronic Medicines Compendium. Jardiance $10 \mathrm{mg}$ and $25 \mathrm{mg}$ film-coated tablets. Summary of Product Characteristics. EMC. www.medicines.org.uk/emc/product/5441/smpc [Accessed 12 November 2020].

13 Zelniker TA, Wiviott SD, Raz I et al. SGLT2 inhibitors for primary and secondary prevention of cardiovascular and renal outcomes in type 2 diabetes: a systematic review and meta-analysis of cardiovascular outcome trials. Lancet 2019;393:31-9.

14 Cannon CP, Pratley R, Dagogo-Jack S et al. Cardiovascular outcomes with ertugliflozin in type 2 diabetes. N Engl J Med 2020;383:1425-35 
15 Heerspink H, Stefansson B, Correa-Rotter R et al. Dapagliflozin in patients with chronic kidney disease. N Engl J Med 2020; 383:1436-46.

16 McMurray J], Solomon SD, Inzucchi SE et al. Dapagliflozin in patients with heart failure and reduced ejection fraction. $N$ Engl J Med 2019:381:1995-2008.

17 Packer M, Anker S, Butler ] et al. Cardiovascular and renal outcomes with empagliflozin in heart failure. $N$ Engl J Med 2020;383:1413-24.

18 Dashora U, Gallagher A, Dhatariya K, Winocour P, Gregory R, ABCD Committee. Association of British Clinical Diabetologists (ABCD) position statement on the risk of diabetic ketoacidosis associated with the use of sodium-glucose cotransporter-2 inhibitors. British Journal of Diabetes 2016;16:206-9.

19 Electronic Medicines Compendium. Steglatro $5 \mathrm{mg}$ and $15 \mathrm{mg}$ filmcoated tablets. Summary of Product Characteristics. EMC. www. medicines.org.uk/emc/product/9803 [Accessed 15 November 2020].

20 Medicines and Healthcare products Regulatory Agency. SGLT2 inhibitors: updated advice on the risk of diabetic ketoacidosis. GOV.UK, 2016. www.gov.uk/drug-safety-update/sglt2-inhibitorsupdated-advice-on-the-risk-of-diabetic-ketoacidosis [Accessed 15 November 2020].

21 European Medicines Agency. SGLT2 inhibitors (previously canagliflozin). EMA, 2017. www.ema.europa.eu/en/medicines/human/ referrals/sglt2-inhibitors-previously-canagliflozin [Accessed 15 November 2020].
22 Medicines and Healthcare products Regulatory Agency. SGLT2 inhibitors: reports of Fournier's gangrene (necrotising fasciitis of the genitalia or perineum). GOV.UK, 2019. www.gov.uk/drugsafety-update/sglt2-inhibitors-reports-of-fournier-s-gangrenenecrotising-fasciitis-of-the-genitalia-or-perineum [Accessed 15 November 2020].

23 Savage MW, Dhatariya KK, Kilvert A et al. Joint British Diabetes Societies guideline for the management of diabetic ketoacidosis. Diabetic medicine 2011;28:508-15.

24 British Cardiovascular Society. Guide for non-diabetes specialist physicians and primary care teams for cardiovascular risk optimisation in patients with Type 2 diabetes and atherosclerotic cardiovascular disease (coronary artery disease, peripheral arterial disease, cerebrovascular disease). BCS, 2020. www.britishcardiovascularsociety.org/_data/assets/pdf_file/0021/21963/CaReMe_T2DM_ CVD_2020.pdf [Accessed 14 January 2021].

Address for correspondence: Dr Umesh Dashora, Diabetes and Endocrinology, Conquest Hospital, East Sussex Healthcare NHS Trust, The Ridge, St Leonards-on-Sea, East Sussex TN37 7RD, UK.

Email: u.dashora@nhs.net

Twitter: @dashorau 